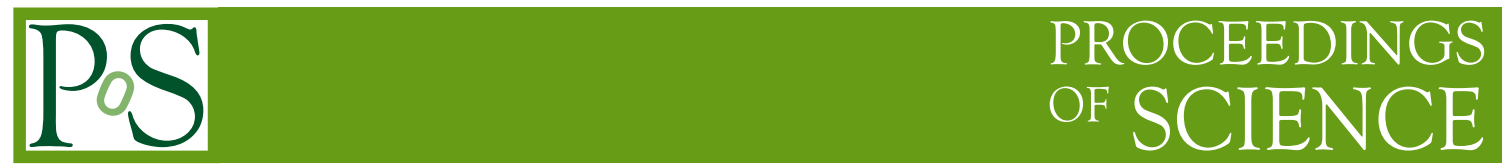

\title{
Boltzmann Boost in Assisted Annihilation
}

\section{Tarak Nath Maity*}

Indian Institute of Technology Kharagpur, Kharagpur-721302, India

E-mail: tarak.maity.physicsegmail.com

\begin{abstract}
Assisted annihilation is a mechanism to generate light dark matter through novel annihilation where two DM and one or more no assister(s) are there in the initial state and two SM or SM-like states are in the final state. We showed that depending on the mass hierarchy between assister and dark matter we can have either a suppression or a boost from a Boltzmann factor in the effective cross section. This boost helps to achieve the required relic density for a $\mathrm{MeV}$ scale dark matter mass.
\end{abstract}

ICHEP 2017, $39^{\text {th }}$ International Conference on High Energy Physics

4-11 July 2018

Seoul, Korea South

${ }^{*}$ Speaker. 


\section{Introduction}

Recently, the dark matter (DM) mass in sub-GeV range with self interaction have drawn considerable attention, for its ability to solve small scale structure formation issues. In our recent work [1] we have introduced the idea of assisted annihilation, where the primary process controlling the relic density is a $n \rightarrow 2$ process. In this article we will prolong our analysis to find new possibilities within the assisted annihilation framework.

\section{Boosted Assisted Annihilation}

The basis constitutes of assisted annihilation framework includes a stable particle $\phi$ which is the DM candidate and assister(s) $A$, which can decay. The typical annihilation processes of this framework take place through the processes where two DM particle and one or more no of assister(s) are there in the initial state and two SM or SM-like particles in the final state. To avoid large flux suppression and to have a cosmologically safe DM, we will confine ourselves to $3 \rightarrow 2$ assisted annihilation processes. The model independent choice of the $3 \rightarrow 2$ thermal average cross section is $\left\langle\sigma v^{2}\right\rangle=g_{1}^{3} / m_{\phi}^{5}$. By solving the relevant Boltzmann equation, we find that depending on mass hierarchy between the assister and the DM either we will have Boltzmann suppression or boost. Since, the equilibrium number density of a non relativistic species falls as $\sim \exp (-m / T)$, therefore, for $m_{\phi}<m_{A}$ small number of assister available to interact with DM. This leads to a suppression in the effective cross section. Thus, in this region of parameter space, assisted annihilation would be an effective mechanism to drive the freeze-out if DM and assister are relatively degenerate. In the figure we have shown the relic density allowed region of parameter space for $m_{\phi}=m_{A} / 2$ by orange line. In this scenario,

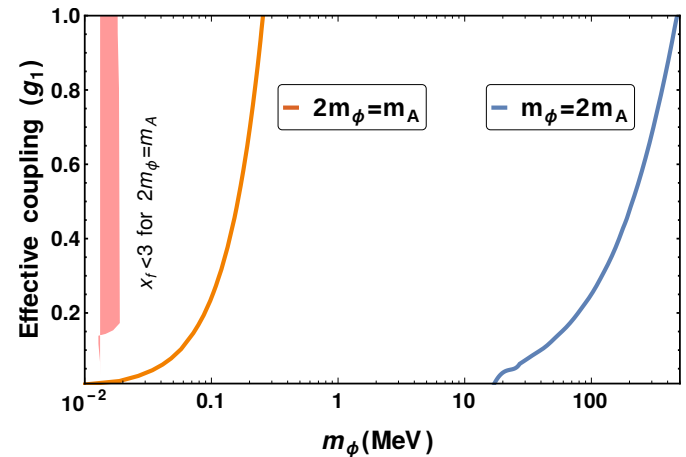

Allowed parameter space in $m_{\phi}-g_{1}$ plane the viable DM mass is in sub-MeV range, which have severe constraint from BBN and CMB observations. However, for $m_{\phi}>m_{A}$, in the thermal soup a large number assister(s) are available to interact with the DM. This will result in a boost in the interaction rate. We will call this boost as Boltzmann boost. We find that this helps to keep viable DM mass in the hundreds of $\mathrm{MeV}$ range. Note that, this is in contrast with usual co-annihilation scenarios where the lightest stable particle is DM, therefore there is no possibility Boltzmann boost. In the figure we have shown the relic density allowed region for $m_{\phi}=2 m_{A}$ by light blue line.

\section{Conclusion}

In this article, we have made phenomenological study of assisted annihilation feasible process to control freeze out. We showed that if the mass of the DM is smaller than the mass of the assister(s) then required relic density can be achieved for a DM mass in sub-MeV region. Whereas, for DM mass greater than assister(s) mass the Boltzmann boost help to have viable DM candidate in the hundred of $\mathrm{MeV}$ range. Presences of such light degrees freedom will manifest in BBN, the detailed study of that is beyond the scope of this article.

\section{References}

[1] U K Dey, T N Maity, T S Ray, JCAP 03 (2017) 045, and references therein. 